\title{
Statistical Analysis of the Tensile Strength of Treated Oil Palm Fiber by Utilisation of Weibull Distribution Model
}

\author{
Chin Voon Sia ${ }^{1,2 *}$, Yoshikazu Nakai ${ }^{1}$, Daiki Shiozawa ${ }^{1}$, Hiroto Ohtani ${ }^{1}$ \\ ${ }^{1}$ Department of Mechanical Engineering, Kobe University, Kobe, Japan; ${ }^{2}$ School of Engineering, Computing and Science, Swinburne \\ University of Technology Sarawak Campus, Kuching, Malaysia. \\ Email: *cvsia@swinburne.edu.my
}

Received December $12^{\text {th }}, 2013$; revised January $7^{\text {th }}$, 2014; accepted January $14^{\text {th }}, 2014$

Copyright (c) 2014 Chin Voon Sia et al. This is an open access article distributed under the Creative Commons Attribution License, which permits unrestricted use, distribution, and reproduction in any medium, provided the original work is properly cited. In accordance of the Creative Commons Attribution License all Copyrights (C) 2014 are reserved for SCIRP and the owner of the intellectual property Chin Voon Sia et al. All Copyright (C) 2014 are guarded by law and by SCIRP as a guardian.

\section{ABSTRACT}

Oil palm fiber (OPF) extracted from empty fruit bunches is proven as a good raw material for bio-composites. The statistical variability in single fiber strength was observed due to the randomly distributed flaws along the fiber. In this study, the effect of gauge length on tensile strength of OPFs has been investigated. The Weibull/ weakest link distribution model was applied to analyse the statistical strength of OPFs. The modified Weibull distribution can predict the gauge length effect more accurately than the conventional Weibull distribution. In addition, the failure strength of the OPF was less sensitive to gauge length compared to coir fiber even though the OPF has a similarity in structures to coir fiber.

\section{KEYWORDS}

Fibers; Strength; Mechanical Testing; Surface Treatment; Gauge Length; Weibull Distribution

\section{Introduction}

Oil palm empty fruit bunch fiber, in short, known as oil palm fiber (OPF) [1] is a kind of seed hair easily to be obtained from oil palm industries [2]. OPFs have not fully materialized in fabrication of biodegradable composites, even though it has the potential to yield up to $73 \%$ fiber at low cost [3]. Therefore, OPF may be more preferable in bio-composites production when considering their availability and cost effectiveness.

The mechanical properties of OPFs are highly variable [1,4-6]. Sia et al. [7] reported that the diameters of natural fibers vary greatly not only among fibers but also along the fiber length. Therefore, statistical approaches to assessing the mechanical properties are essential to analyse the high disparity of the mechanical properties and to quantify the variation of the probabilistic strength of OPFs. Recently, the Weibull/weakest link distribution [8] has been widely applied to the statistical analysis of mechanical strengths of natural fiber such as abaca [9], jute [10], okra [11] and flax [12].
The common two-parameter Weibull distribution applied in the mechanical study is given by:

$$
F=1-\exp \left[-\frac{V}{V_{0}}\left(\frac{\sigma}{\sigma_{0}}\right)^{m}\right]
$$

where $F$ is the failure probability of the fiber, $V$ is fiber volume, $\sigma$ is failure strength, $V_{0}$ is standard volume, $m$ is Weibull modulus and $\sigma_{0}$ is scale parameter.

The average value of $\sigma$ can be predicted from the equation below once the Weibull distribution parameters $(m$, $\sigma_{0}$ ) are obtained:

$$
\bar{\sigma}=\sigma_{0}\left(\frac{V}{V_{0}}\right)^{-1 / m} \Gamma\left(1+\frac{1}{m}\right)
$$

For a constant fiber diameter, when the average value of $\sigma_{1}$ at gauge length $L_{1}$ and $m$ can be obtained, the average value of $\sigma_{2}$ at the gauge length $L_{2}$ can be calculated:

$$
\bar{\sigma}_{2}=\bar{\sigma}_{1}\left(\frac{L_{2}}{L_{1}}\right)^{-1 / m}
$$


where the values of $\sigma_{1}$ and $\sigma_{2}$ are the fiber stress at gauge length $L_{1}$ and gauge length $L_{2}$ respectively.

However, great discrepancies were found between conventional Weibull predicted strength and experimental data $[13,14]$. Therefore, Watson and Smith [15] as well as Gatans and Tamuzs [16] have suggested that the exponent $1 / m$ in Equation (3) be modified to $\alpha / m$ :

$$
\bar{\sigma}_{2}=\bar{\sigma}_{1}\left(\frac{L_{2}}{L_{1}}\right)^{-\alpha / m}, 0<\alpha<1
$$

and the corresponding Weibull distribution (named as WSGT function [17]) becomes:

$$
F=1-\exp \left[-\left(\frac{V}{V_{0}}\right)^{\alpha}\left(\frac{\sigma}{\sigma_{0}}\right)^{m}\right], 0<\alpha<1
$$

Manipulation above expression gives the following relation:

$$
\ln (-\ln (1-F))-\alpha \ln \frac{V}{V_{0}}=m \ln \sigma-m \ln \sigma_{0}
$$

Hence, a plot of $\ln (-\ln (1-F))-\alpha \ln \frac{V}{V_{0}}$ versus $\ln \sigma$, which should produce a straight line, gives rise to the gradient, $m$ and intercepts $\sigma_{0}$ at $\ln (-\ln (1-F))-\alpha \ln \frac{V}{V_{0}}=0$. Median rank method is usually used as an estimator for probability of fiber failure.

$$
F=\frac{i-0.3}{N+0.4}
$$

where $i$ is the rank of the each data point and $N$ is the total number of the samples.

The parameter of $\alpha$ was found to vary in previous researches. Phoenix et al. [18] suggested that $\alpha=0.60$ for Kevlar-49 fibers, and Wu and Netravali [19] obtained $\alpha=$ 0.77 for Nicalon SiC fibers.

However, Zhang et al. [20] suggested that the value of $\alpha$ in Equation (4) is related to the within-fiber diameter variation:

$$
\ln \left(\mathrm{CV}_{\mathrm{FD}}\right)=\alpha \ln (L)+A+\varepsilon
$$

where $\mathrm{CV}_{\mathrm{FD}}$ is the average within-fiber diameter variation, $L$ is the gauge length, $A$ is a constant, $\varepsilon$ is a random error.

Considering all the above issues, it is important to determine a suitable Weibull model to predict the tensile strength of OPFs and other natural fibers which have the similar structures. The comparison of the statistical analysis by using the conventional Weibull distribution and the modified Weibull model is also described in this paper.

\section{Experimental}

\subsection{Materials}

Oil palm fibers were purchased from CM Fibre Processing Sdn Bhd, Malaysia. The average fiber diameters used in this experiment is in between $190 \mu \mathrm{m}$ to $300 \mu \mathrm{m}$. Sodium Hydroxide $(\mathrm{NaOH})$ was purchased in Hyogo, Japan.

\subsection{Surface Treatment}

Oil palm fibers were kept in an oven at $21^{\circ} \mathrm{C} \pm 1^{\circ} \mathrm{C}$ for 24 hours for conditioning which accords with ASTM D1776-04. The oil palm fibers were then soaked in $1.0 \mathrm{M}$ of alkali solution for 24 hours. After completing the surface treatment, the alkali treated fibers were washed and rinsed with distilled water several times until the final $\mathrm{pH}$ was maintained at 7.0. Finally, the fibers were dried at room temperature for 48 hours.

\subsection{Fiber Diameter Scanning}

The specimens were prepared according to the ASTM C1557-03. The fiber was mounted on a slotted cardboard tabas shown in Figure 1. Extreme care was taken to ensure that the fiber was aligned axially with the tensile direction. Cemedine EP001N adhesive was used to attach the fiber at opposite ends of slot. The gauge length was determined by the fiber length between the adhesive spots. Once the glue spots were cured, the fibers were placed under a Nikon Eclipse ME600 microscope for diameter scanning. The fibers were considered perfectly round and the fiber diameters were measured at $1 \mathrm{~mm}$ intervals along its length.

\subsection{Tensile Test}

The uniaxial tensile strength tests were carried out by using a Tohei MT201 tensile test machine. The tensile strengths of the OPFs were evaluated at $10 \mathrm{~mm}, 20 \mathrm{~mm}$, $30 \mathrm{~mm}$ and $40 \mathrm{~mm}$ gauge lengths. The values for the fiber fractures at or near the adhesive spots were discarded. Fifty sets of successful data at each of these gauge lengths were obtained for the statistical analysis.

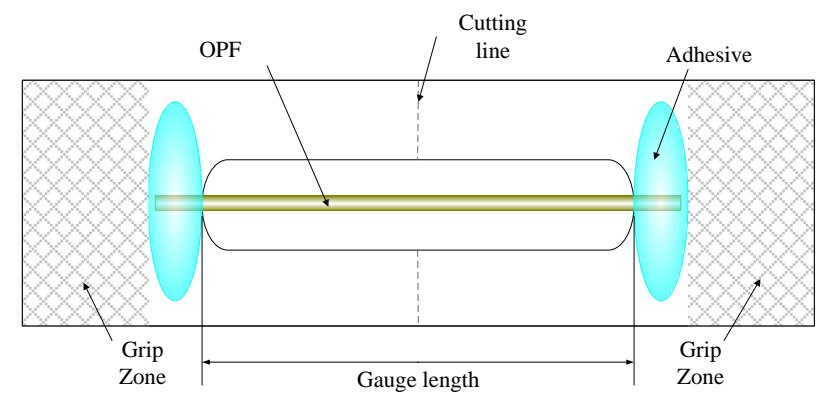

Figure 1. Schematic of the specimen. 


\section{Results and Discussion}

\subsection{Morphological Analysis}

The micrographs of the fiber before and after alkali treatment are exhibited in Figures 2(a) and (b) respectively. $\mathrm{NaOH}$ treatment leads to the irreversible mercerization by removing natural and artificial impurities, thereby producing clean and more visible fibrils on surface topography (Figure 2(b)). Many studies also revealed that the impurities in natural fibers can be washed out by alkali treatment [21,22]. In this study, the parameter $\alpha$ is dependent on the within-fiber diameter variation along the fiber length. Therefore, a clean fiber surface can improve the accuracy of the statistical analysis.

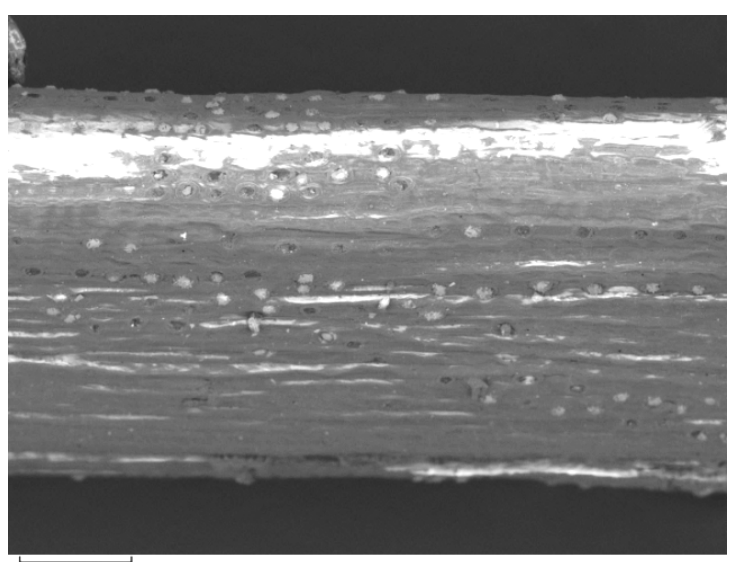

$$
100 \mu \mathrm{m}
$$

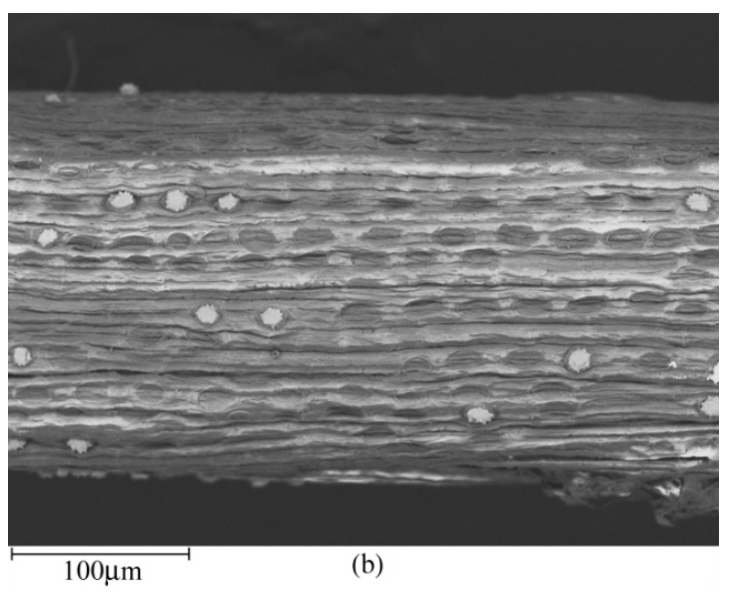

Figure 2. SEM micrographs of the surface of oil palm fiber. (a) Untreated fiber; (b) Treated fiber.

\subsection{Weibull Distribution}

The mean diameter listed in Table 1 is the average diameter of 50 tested fibers. The $\mathrm{CV}_{\mathrm{FD}}$ is the average value of the within-fiber diameter variation coefficients of 50 fibers at each gauge length. The average value of failure strength decreases with increasing in gauge length is probably due to the fact that the flaw sizes increase in larger fiber volume. Similar behaviour is observed in wool [20], banana [23] and coir [24] fibers. The relationship between $C V_{F D}$ and the gauge length of the test specimens is illustrated in Figure 3. The average withinfiber diameter variation increases exponentially with the increase of the gauge length. The correlation of the logarithm in Figure 3 also shows a very high value $\left(R^{2}=\right.$ 0.9829). As reported by Zhang et al. [20], the value $\alpha=$ 0.2133 can be obtained from the slope value of $\ln \left(\mathrm{CV}_{\mathrm{FD}}\right)$ versus $\ln (L)$.

The Weibull plots based on Equation (6) for the OPF failure stress at $10 \mathrm{~mm}, 20 \mathrm{~mm}, 30 \mathrm{~mm}$ and $40 \mathrm{~mm}$ gauge lengths are illustrated in Figure 4. The linear regression of the failure strength at all gauge lengths shows a good approximation to experiment data where the value of $R^{2}$ is from $95.92 \%$ to $98.98 \%$. The Weibull modulus, $m$ and the scale parameter, $\sigma_{0}$ obtained from Figure 4 at gauge lengths of $10 \mathrm{~mm}, 20 \mathrm{~mm}, 30 \mathrm{~mm}$ and $40 \mathrm{~mm}$ are respectively listed in Table 1.

\subsection{Strength Prediction of the Fiber}

The Weibull model can predict the gauge length effect on the fiber strength if the data fit the distribution well. The

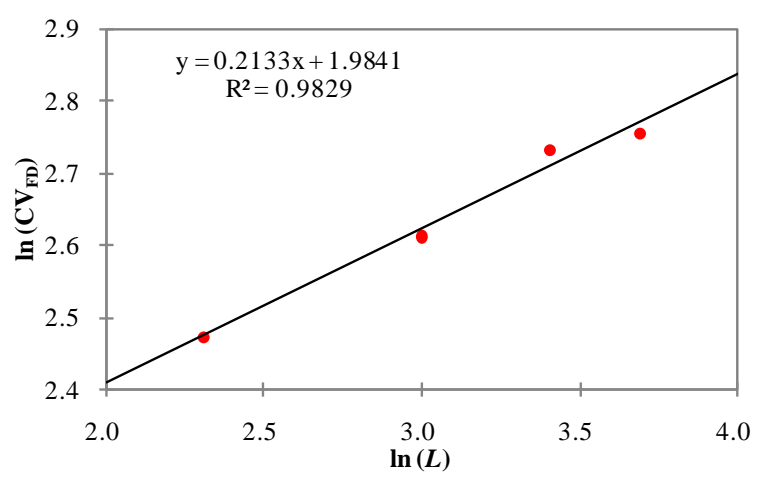

Figure 3. The relationship between $\mathrm{CV}_{\mathrm{FD}}$ and the gauge length.

Table 1. Mean diameter, $\mathbf{C V}_{\mathrm{FD}}$, failure strength and Weibull parameters of fibers at various gauge lengths.

\begin{tabular}{cccccc}
\hline Gauge length, $L(\mathrm{~mm})$ & Mean diameter, $\bar{D}_{\mathrm{f}}(\mu \mathrm{m}) \mathrm{CV}_{\mathrm{FD}}$ within the fiber (\%) Failure Strength, $\sigma_{\mathrm{f}}(\mathrm{MPa})^{\mathrm{a}}$ Weibull modulus, $m$ Scale Parameter, $\sigma_{0}$ \\
\hline 10 & 245.30 & 11.90 & $177.68 \pm 38.41$ & 5.47 & 192.35 \\
20 & 240.08 & 13.62 & $170.89 \pm 35.78$ & 5.60 & 184.10 \\
30 & 245.60 & 15.38 & $161.48 \pm 37.71$ & 4.82 & 176.23 \\
40 & 247.80 & 15.77 & $160.28 \pm 37.66$ & 4.95 & 174.75 \\
\hline
\end{tabular}

${ }^{\mathrm{a}}$ Mean \pm standard deviation based on fifty samples. 

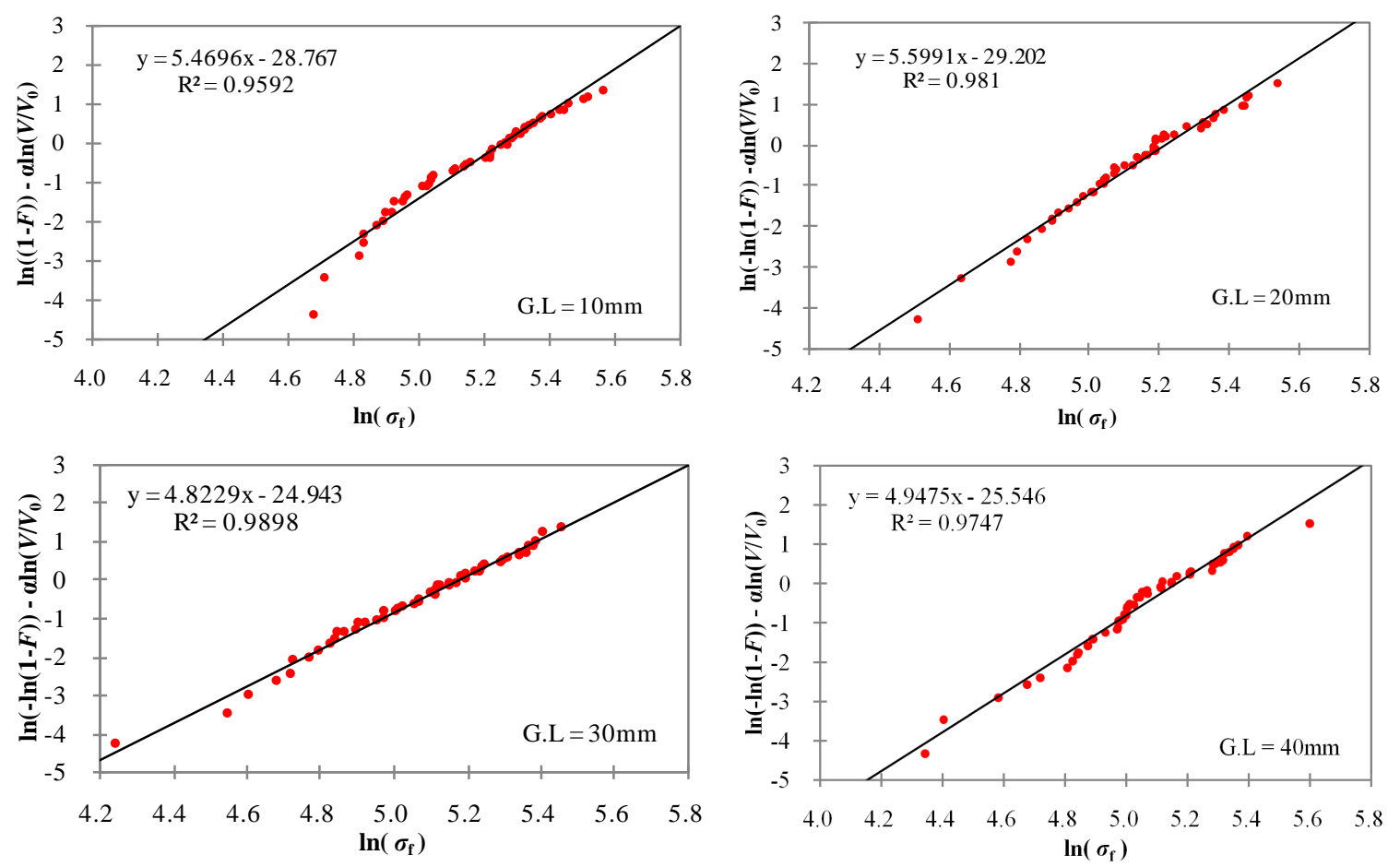

Figure 4. The Weibull plots for the failure strength of oil palm fibers at each gauge length.

predicted failure strength from the conventional Weibull distribution and the modified Weibull distribution based on the experimental value at $10 \mathrm{~mm}$ and $40 \mathrm{~mm}$ are shown in Figures 5(a) and (b) respectively. The disparity between the experimental data and the conventional Weibull predicted failure strength was observed in Figures 5(a) and (b). The failure strength predicted by the modified Weibull model is more accurate than the conventional Weibull distribution, where the modified Weibull predicted strength lies within 95\% confidence intervals of the experimental results.

\subsection{The Parameter $\alpha$ in Predicted Strength}

A comparison between the experimental data and predicted values from Equation (4) at different value of parameter $\alpha$ is shown in Figure 6. The predicted curve with the parameter $\alpha=0.4$ was found to have a good agreement with experimental values. However, the parameter $\alpha=0.4$ is specifically significant at very short gauge length. Applying the parameter $\alpha=0.4$ at long gauge length may decrease the predictive accuracy.

\subsection{Scale Effect}

The failure strength of banana [23], coir [24], ramie [25] and kenaf [25] fibers at various gauge lengths are provided in Table 2. As expected, the failure strength of banana, coir, ramie and kenaf fibers decrease with increasing in gauge length.

The plot of normalised failure strength at different

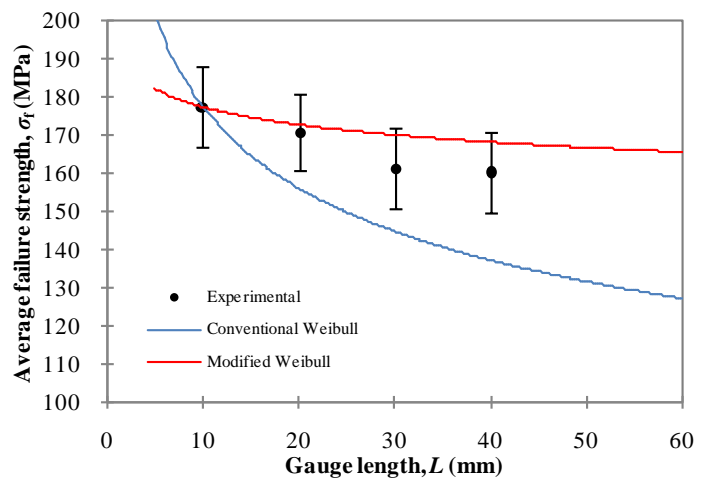

(a)

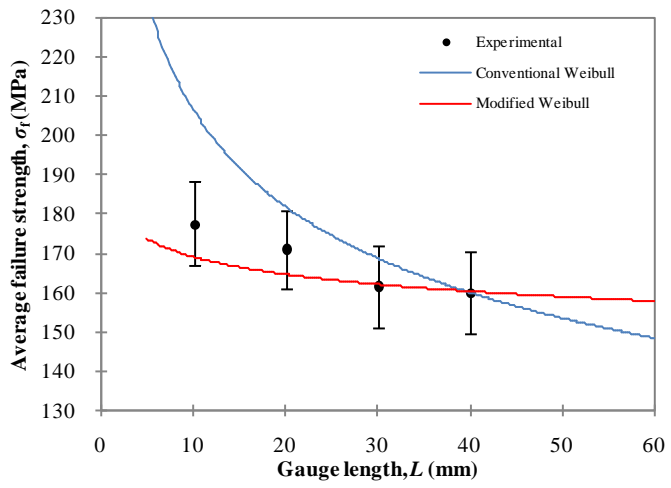

(b)

Figure 5. Comparison of average fiber failure strength between experimental and predicted values based on the experimental data at (a) $10 \mathrm{~mm}$ and (b) $40 \mathrm{~mm}$ gauge length (Experimental results are plotted at $95 \%$ confidence intervals). 


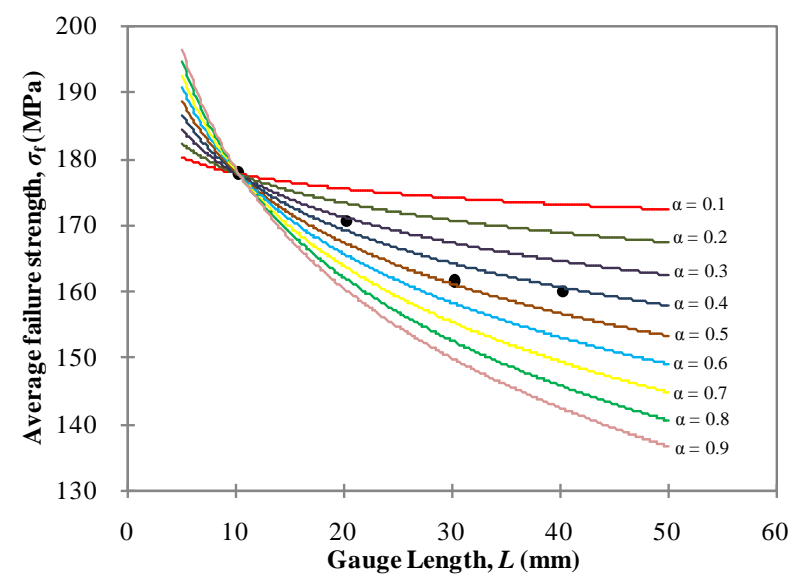

Figure 6. Comparison between the experimental and the predicted values of average failure strength of oil palm fibers at different value of parameter $\alpha$ (prediction based on the result at $10 \mathrm{~mm}$ gauge length).

Table 2. Failure strength of natural fibers at various gauge lengths.

\begin{tabular}{ccccc}
\hline \multirow{2}{*}{$\begin{array}{c}\text { Gauge length, } L \\
(\mathrm{~mm})\end{array}$} & \multicolumn{4}{c}{ Fiber failure strength, $\sigma_{\mathrm{f}}(\mathrm{MPa})$} \\
\cline { 2 - 5 } & Banana [23] $^{\mathrm{a}}$ & ${\text { Coir }[24]^{\mathrm{a}}}^{\mathrm{R}}$ & Ramie [25] & Kenaf [25] \\
\hline 10 & 1055.516 & 279.17 & 2517 & 1137 \\
20 & 930.734 & 265.98 & 2380 & 1010 \\
35 & 891.706 & 211.15 & - & - \\
50 & 711.661 & 175.62 & 2172 & 888 \\
\hline
\end{tabular}

${ }^{\mathrm{a}}$ References.

gauge lengths is illustrated in Figure 7. The strength values of oil palm, banana, coir, ramie and kenaf fibers are normalized by using each respective strength value at $10 \mathrm{~mm}$. Clearly, the OPF and ramie fiber are less sensitive to its gauge length compared to kenaf, banana and coir fibers. The scale effect of the fiber strength depends on the growth rate of the flaw sizes in the fiber when the gauge length is increased.

As seen from Figure 7, the failure strength of the OPF has less scale effect on its gauge length compared to coir fiber although Hassan et al. [26] have reported that the OPF has a similarity in structures to coir fiber. This could be due to the growth rate of the flaw sizes and the flaw distributions differ between OPF and coir fiber.

\section{Conclusions}

The present work evaluated the failure strength of the OPFs at different gauge lengths. A modified Weibull statistical analysis has been addressed. The following conclusions can be drawn from this work:

1) The average failure strength of the OPF increases with decreasing in gauge length because the flaw sizes increase in larger fiber volume.

2) The predicted fiber strength by the modified Weibull model is more accurate than the conventional Wei-

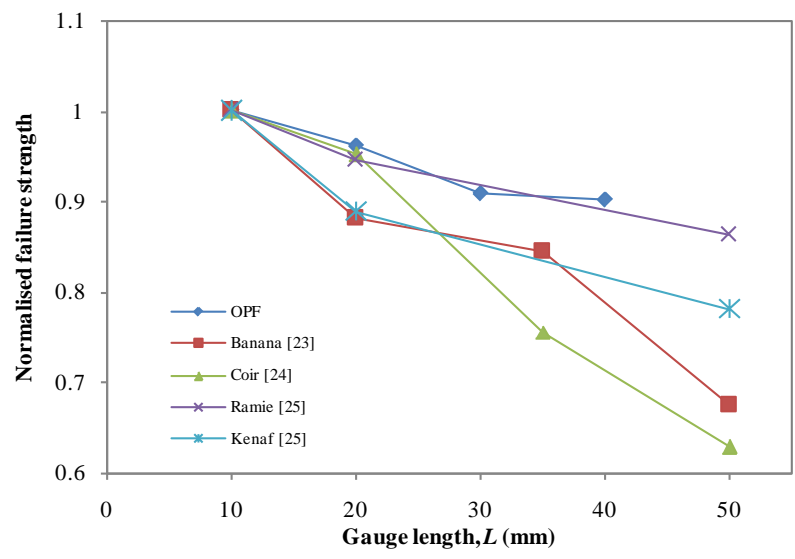

Figure 7. Normalised failure strength of oil palm, banana, coir, ramie and kenaf fibers at various gauge lengths.

bull distribution. The statistical analysis of OPF strength by incorporating the within-fiber diameter variation should be considered.

3) The optimum value of the parameter $\alpha=0.4$ was obtained in this study. However, the value of the parameter $\alpha$ is specifically significant at very short gauge length.

4) The scale effect may vary although the fibers have a similarity in structures. This may be due to the growth rate of the flaw sizes and the flaw distributions differing from fiber to fiber.

\section{REFERENCES}

[1] S. Shinoj, R. Visvanathan, S. Panigrahi and M. Kochubabu, "Oil Palm Fiber (OPF) and Its Composite: A Review,” Journal of Industrial Crops and Products, Vol. 33, No. 1, 2011, pp. 7-22.

http://dx.doi.org/10.1016/j.indcrop.2010.09.009

[2] B. Wirjosentono, P. Guritno and H. Ismail, "Oil Palm Empty Fruit Bunch Filled Polypropylene Composite,” International Journal of Polymeric Materials, Vol. 53, No. 4, 2004, pp. 295-306.

http://dx.doi.org/10.1080/00914030490429942

[3] H. D. Roszman, C. Y. Lai, H. Ismail and Z. A. M. Ishak, "The Effect of Coupling Agents on the Mechanical and Physical Properties of Oil Palm Empty Fruit Bunch-Polypropylene Composites,” Polymer International, Vol. 49, No. 11, 2000, pp. 1273-1278.

http://dx.doi.org/10.1002/1097-0126(200011)49:11<1273 :AID-PI469>3.0.CO;2-U

[4] M. S. Sreekala, M. G. Kumaran, M. L. Geethakumariamma and S. Thomas, "Environmental Effects in Oil Palm Fiber Reinforced Phenol Formaldehyde Composites," Composite Part A, Vol. 33, No. 6, 2004, pp. 763-777. http://dx.doi.org/10.1016/S1359-835X(02)00032-5

[5] A. Kalam, B. B. Sahari, Y. A. Khalid and S. V. Wong, "Fatigue Behaviour of Oil Palm Empty Fruit Bunch Fiber/Epoxy and Carbon Fiber/Epoxy Composites," Composite Structures, Vol. 71, No. 1, 2005, pp. 34-44. 
http://dx.doi.org/10.1016/j.compstruct.2004.09.034

[6] B. F. Yousif and E. N. S. M. Tayeb, "The Effect of Oil Palm Fibers as Reinforcement on Tribological Performance of Polyester Composite," Surface Review and Letters, Vol. 14, No. 6, 2007, pp. 1095-1102. http://dx.doi.org/10.1142/S0218625X07010561

[7] C. Sia, Y. Nakai and D. Shiozawa, "Influence of Alkali Treatment on the Mechanical Properties of Oil Palm Fibers," Proceeding of 4th Japan Conference on Composite Materials, Tokyo, 7-9 March 2013.

[8] W. Weibull, "A Statistical Distribution Function of Wide Applicability,” ASME Journal of Applied Mechanics, Vol. 18, 1951, pp. 293-297.

[9] L. Peponi, J. Biagiotti, L. Torre, J. M. Kenny and I. Mondragon, "Statistical Analysis of the Mechanical Properties of Natural Fibers and Their Composite Materials. I. Natural Fiber," Polymer Composites, Vol. 29, No. 3, 2008, pp. 313-320. http://dx.doi.org/10.1002/pc.20408

[10] A. Roy, S. Chakraborty, S. P. Kundu, R. K. Basak, S. B. Majumder and B. Adhikari, "Improvement in Mechanical Properties of Jute Fibres through Mild Alkali Treatment as Demonstrated by Utilisation of the Weibull Distribution Model,” Bioresource Technology, Vol. 107, 2011, pp. 222-228. http://dx.doi.org/10.1016/j.biortech.2011.11.073

[11] I. M. Rosa, J. M. Kenny, D. Puglia, C. Santulli and F. Sarasini, "Morphological, Thermal and Mechanical Characterization of Okra (Abelmoschus esculentus) Fibres as Potential Reinforcement in Polymer Composites," Composites Science and Technology, Vol. 70, No. 1, 2010, pp 116-122. http://dx.doi.org/10.1016/j.compscitech.2009.09.013

[12] J. Andersons, E. Sparnins, R. Joffe and L. Wallstrom, "Strength Distribution of Elementary Flax Fibres," Composites Science and Technology, Vol. 65, No. 3-4, 2005, pp. 693-702.

http://dx.doi.org/10.1016/j.compscitech.2004.10.001

[13] K. L. Pickering, G. W. Beckermann, S. N. Alam and N. J. Foreman, "Optimising Industrial Hemp Fibre for Composites,” Composites Part A, Vol. 38, No. 2, 2007, pp. 461468. http://dx.doi.org/10.1016/j.compositesa.2006.02.020

[14] D. M. Wilson, "Statistical Tensile Strength of Nextel ${ }^{\mathrm{TM}}$ 610 and Nextel ${ }^{\mathrm{TM}} 720$ Fibres," Journal of Materials Science, Vol. 32, No. 10, 1997, pp. 2535-2542. http://dx.doi.org/10.1023/A:1018538030985

[15] A. S. Watson and R. L. Smith, “An Examination of Statistical Theories for Fibrous Materials in the Light of Experimental Data,” Journal of Materials Science, Vol. 20, No. 9, 1985, pp. 3260-3270. http://dx.doi.org/10.1007/BF00545193

[16] J. A. Gutans and V. P. Tamuzh, "Scale Effect of the Weibull Distribution of Fibre Strength,” Mechanical Compo- site Materials, Vol. 6, 1984, pp. 1107-1109.

[17] H. D. Wagner, "Statistical Concepts in the Study of Fracture Properties of Fibers and Composites," Application of Fracture Mechanics to Composite Materials, Vol. 6, 1989, pp. 39-67.

[18] S. L. Pheonix, P. Schwartz and H. H. Robinson IV, "Statistics for the Strength and Lifetime in Creep-Rupture of Model Carbon/Epoxy Composites," Composite Science and Technology, Vol. 32, No. 2, 1988, pp. 81-120. http://dx.doi.org/10.1016/0266-3538(88)90001-2

[19] H. F. Wu and A. N. Netravali, "Weibull Analysis of Strength-Length Relationships in Single Nicalonsicfibres," Journal of Materials Science, Vol. 27, No. 12, 1992, pp. 3318-3324. http://dx.doi.org/10.1007/BF01116031

[20] Y. Zhang, X. Wang, N. Pan and R. Postle, "Weibull Analysis of the Tensile Behavior of Fibers with Geometrical Irregularities,” Journal of Materials Science, Vol. 37, No. 7, 2002, pp. 1401-1406. http://dx.doi.org/10.1023/A:1014580814803

[21] M. S. Sreekala, M. G. Kumaran, R. Joseph and S. Thomas, "Stress-Relaxation Behaviour in Composites Based on Short Oil-Palm Fibres and Phenol Formaldehyde Resin,” Composites Science and Technology, Vol. 61, No. 9, 2001, pp. 1175-1188. http://dx.doi.org/10.1016/S0266-3538(00)00214-1

[22] A. K. M. Moshiul Alam, D. M. Reddy Prasad, M. R. Khan and M. F. Mina, "Structures and Performance of Simultaneous Ultrasound and Alkali Treated Oil Palm Empty Fruit Bunch Fiber Reinforced Poly(Lactic Acid) Composites," Composites Part A, Vol. 43, No. 11, 2012, pp. 1921-1929. http://dx.doi.org/10.1016/S0266-3538(00)00214-1

[23] A. G. Kulkarni, K. G. Satyanarayana and P. K. Rohatgi, "Mechanical Properties of Banana Fibres," Journal of Materials Science, Vol. 18, No. 8, 1983, pp. 2290-2296. http://dx.doi.org/10.1007/BF00541832

[24] A. G. Kulkarni, K. G. Satyanarayana and P. K. Rohatgi, "Mechanical Behaviour of Coir Fibres under Tensile Load,” Journal of Materials Science, Vol. 16, No. 4, 1981, pp. 905-914. http://dx.doi.org/10.1007/BF00542734

[25] J. M. Park, T. Q. Son, J. G. Jung, S. J. Kim and B. S. Hwang, "Interfacial Evaluation of Single Ramie and Kenaf Fibers/Epoxy Composites Using Micromechanical Techniques,” Journal of Adhesion and Interface, Vol. 6, No. 2, 2005, pp. 13-20.

[26] A. Hassan, A. A. Salema, F. N. Ani and A. A. Bakar, “A Review on Oil Palm Empty Fruit Bunch Fiber-Reinforced Composite Materials,” Polymer Composites, Vol. 31, No. 12, 2010, pp. 2079-2101. http://dx.doi.org/10.1002/pc.21006 